\title{
Synthesis of CuO powders using different fuels for selective surfaces
}

María Celeste Gardey Merino ${ }^{1}$, José Antonio Alonso ${ }^{2}$, Patricia Vázquez ${ }^{3}$, Valeria Palermo ${ }^{3}$, Silvina Lassa ${ }^{4}$, Mariana Rodriguez ${ }^{1}$, Ariel Bordón ${ }^{1}$

${ }^{1}$ Grupo CLIOPE-UTN Regional Mendoza, Ciudad De Mendoza, Argentina, ${ }^{2}$ Instituto de Ciencia de Materiales de Madrid (CSIC), Madrid, Spain, ${ }^{3}$ CINDECA, CCT CONICET - La Plata, La Plata, Argentina, ${ }^{4}$ MEByM - IANIGLA CONICET-Mendoza,, Mendoza, Argentina E-mail: mcgardey@frm.utn.edu.ar

Cupric oxide ( $\mathrm{CuO})$ is used as absorbent film in solar selective surfaces. CuO nanostructures, in particular, are promising materials for the fabrication of solar cells because of their high solar absorbance, low thermal emittance, relatively good electrical properties, and high carrier concentration. The methods used for obtain (CuO) nanostructures are: hydrothermal, precipitation, solid-state thermal conversion of precursors, electrochemical, thermal oxidation [1] and by urea-nitrates combustion synthesis [2]. This work is aimed to the production of $\mathrm{Cu}$ oxides by means of original one-step stoichiometric combustion methods from Cu(NO3)2.3H2O and aspartic acid (Asp), lysine (Lys), ethylene diamine tetra-acetic acid (EDTA) or tetrametilaminomethane (TRIS) as fuels. Once obtained by combustion processes, the ashes were calcined at $500^{\circ} \mathrm{C}$ in order to get the pigments with the desired crystalline structure. The powders were characterized by $\mathrm{X}$-ray diffraction (XRD), scanning electron microscopy (SEM), transmission electron microscopy (TEM), and the crystal structures were refined by the Rietveld method. The refinement of the crystal structure allowed us demonstrating the single-phase nature of the product and its crystallographic features. This is of importance to associate the properties of the material with the oxygen coordination and oxidation state of the $\mathrm{Cu}$ cations. The diffractograms of the all obtained powders correspond to CuO, with tenorite crystalline structure, defined in the monoclinic $\mathrm{C} 2 / \mathrm{c}$ space group (No. 15$)$, origin at center (2/m). For example for Tris-calcined powders, the unit-cell parameters are the following: $a=4.687(1) \AA, b=3.427(1) \AA, c=5.133(1) \AA$, beta= $99.36(1)^{\circ}$. In the crystal structure, $\mathrm{Cu}$ was located at $4 \mathrm{c}(1 / 4,1 / 4,0)$ and $\mathrm{O}$ at $4 \mathrm{e}(0,0.4264,1 / 4)$ crystallographic Wyckoff sites. A good agreement factors is obtained for this model. An excellent fit was obtained between observed and calculated XRD profiles, as shown in Fig.1, attaining RBragg $=3.64 \%$ for the tris-calcined powders. The unit-cell parameters for CuO obtained from different fuels present significant differences, probably due to the different nature of the fuels that implies different reaction enthalpies. Structurally, the distinct $\mathrm{Cu}-\mathrm{O}$ distances may be ascribed to slight variations in the $\mathrm{Cu}$ oxidation state, caused by subtle oxygen non-stoichiometry that cannot be assessed from a XDR study. The average crystallite size calculated by the Scherrer equation ranged between 29 and $51 \mathrm{~nm}$ for the calcined powders. By SEM, it can be observed that all the samples exhibit a high degree of agglomeration. As estimated through TEM, the particle size ranges from 20 to $200 \mathrm{~nm}$.

[1] Qiaobao Zhang et al. (2014). Prog. Mater. Sci. 60, 208-337.

[2] Hossein Ajameina et al. (2016). J. Mol. Catal. A: Chem. 421, 222-234.

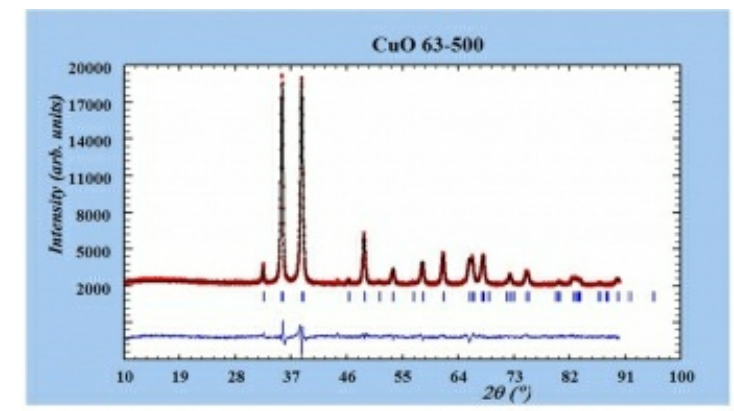

Figure 1. Rietveld analysis of Tris-calcined-powder sample.

Keywords: Keywords: $\mathrm{CuO}$, combustion synthesis, Rietveld Analysis 